\title{
La ventilation mécanique aux urgences : promouvoir une compétence du médecin urgentiste adaptée aux profils des patients
}

\author{
Mechanical ventilation in emergency department: \\ Developing competencies of emergency physicians according to the needs of patients
}

\author{
F. Templier \\ Reçu le 23 juin 2011 ; accepté le 24 juin 2011 \\ (C) SFMU et Springer-Verlag France 2011
}

« La ventilation mécanique (VM) invasive ou non invasive est une pratique quotidienne en médecine d'urgence, dans le contexte préhospitalier comme à l'accueil. » Cette affirmation ne semble pas choquante. Et pourtant, quel est notre niveau réel de connaissance dans ce domaine ? Plusieurs études ont démontré l'intérêt sous certaines conditions de la ventilation non invasive (VNI) dans les structures d'urgences, et des recommandations dans ce contexte spécifique ont pu être établies [1]. Toutefois, peu d'études ont décrit les pratiques quotidiennes [2]. Paradoxalement, bien que d'usage plus ancien que la VNI, la littérature sur la ventilation mécanique invasive (VI) aux urgences est d'une très grande pauvreté. Nous n'avons pas de vision précise de l'incidence de la VM aux urgences et sa durée, des profils des patients ventilés, du type de matériel et des modes ventilatoires utilisés...

Ce numéro des Annales françaises de médecine d'urgence publie les résultats du travail mené par Roti et al. [3], dont l'objectif était de décrire les caractéristiques principales des patients ventilés (VI ou VNI) au sein de deux structures d'urgences d'un même centre hospitalier. Quatre grands messages s'en dégagent :

- la VM est bien une pratique quotidienne aux urgences, quatre fois plus souvent en VI qu'en VNI. La médiane de durée de ventilation y est de quelques heures ;

- les objectifs de ventilation et les conditions pulmonaires des patients varient. En VI, un tiers des patients ont une souffrance cérébrale aiguë possible, nécessitant un contrôle strict de la $\mathrm{PaCO}_{2}$, et presque un quart ont des conditions pulmonaires anormales (obstructif, restrictif,

F. Templier $(\varangle)$

Samu 49-Smur Angers, centre hospitalier universitaire d'Angers, avenue de l'Hôtel-Dieu, F-49933 Angers cedex 09, France e-mail : FrTemplier@chu-angers.fr mixte, compliance basse), pouvant rendre difficile l'adaptation de la ventilation;

- les urgentistes privilégient les ventilateurs performants plutôt que les ventilateurs de transport plus basiques. En VI, le mode ventilation contrôlée (VC) stricte est plus souvent utilisé que le mode ventilation assistée contrôlée (VAC). L'alternative est l'usage du mode adaptative support ventilation (ASV), présent sur un des ventilateurs disponibles ;

- que le recours à la VNI aux urgences dans la pratique de tous les jours est possible.

Bien sûr, le caractère " juste » bicentrique de ce travail expose inévitablement au biais classique de "l'effet centre », notamment sur l'utilisation de l'ASV. Il ne répond pas non plus à toutes les questions qui se posent sur cette thématique : sur quels critères ont été choisis les ventilateurs et les réglages, et notamment pourquoi régler en $\mathrm{VC}$ plutôt qu'en VAC ? Quel en a été le résultat sur l'hématose ? Quelles ont été les difficultés d'adaptation patient-ventilateur? Quels sont les événements indésirables liés à la ventilation, avec quelles conséquences pour le patient ? Comment les cliniciens se sentaient-ils préparés pour gérer des situations de ventilation très diverses ? Mais ne gâchons pas ici notre plaisir de voir abordée enfin cette problématique de la VM aux urgences, et en plus publiée dans un des premiers numéros de la revue d'expression scientifique de notre Société, la Sfmu. C'est un signal en faveur de l'intérêt que doit porter notre « spécialité » de médecine d'urgence vis-à-vis de la VM sous toutes ses formes. Il doit nous inciter à poursuivre la réflexion dans trois grands axes :

- quelle doit être la compétence des urgentistes pour un usage optimal de la VM? La description des profils des patients ventilés aux urgences est une étape préalable incontournable. Cette compétence n'a sans doute pas besoin d'être aussi poussée que celle d'un réanimateur. 
Probablement... quoique l'urgentiste, en structure d'accueil un jour, transférera en Smur un autre jour un patient présentant un syndrome de détresse respiratoire aiguë difficilement ventilable. À l'évidence, l'urgentiste ne doit plus se contenter de savoir tout juste utiliser un simple ventilateur pneumatique de première ou deuxième génération;

- quel est l'impact de nos pratiques sur le devenir de nos patients et comment pouvons-nous améliorer celles-ci ? L'évaluation, notamment dans le cadre de l'EPP, doit être largement encouragée. Des travaux de recherche clinique doivent également être menés pour déterminer l'impact clinique de telle ou telle stratégie de ventilation selon les différents profils des patients ;

- quels sont les types de ventilateurs qui doivent être disponibles dans nos structures d'urgences? Les propositions avancées lors des journées scientifiques de Samu de France sur la dyspnée aiguë en 2004 pour le contexte préhospitalier pourraient servir de base à cette réflexion [4].

Enfin, ne perdons jamais de vue que la médecine d'urgence hospitalière et préhospitalière s'adresse aux mêmes patients, et qu'elle est pratiquée par les mêmes médecins. L'amélioration de nos pratiques aux urgences aura un impact en Smur et inversement. Et sans jamais oublier que notre spécialité s'exerce en équipe, et que tous les autres soignants doivent être intégrés de façon active à la démarche médicale d'amélioration de la pratique de la ventilation en médecine d'urgence.

Conflit d'intérêt : l'auteur déclare ne pas avoir de conflit d'intérêt.

\section{Références}

1. Robert R, Bengler C, Beuret P, et al (2006) Ventilation non invasive au cours de l'insuffisance respiratoire aiguë (nouveau-né exclu), texte long du jury. Conférence de consensus commune de la SRLF, de la SFAR et de la SPLF : 5-12 (Available online at www.sciencedirect.com)

2. Vanpee D, Delaunois L, Lheureux P, et al (2002) Survey of non-invasive ventilation for acute exacerbation of chronic obstructive pulmonary disease patients in emergency department in Belgium. Eur J Emerg Med 9:217-24

3. Roti M, Arnal JM, Delnista D, et al (2011) Étude prospective observationnelle bicentrique sur la pratique de la ventilation mécanique aux urgences 1:305-11

4. Templier F, Thys F, Durand JS, Jardel B (2005) Oxygénothérapie et supports ventilatoires. In: Dyspnée aiguë. Journées scientifiques de Samu de France 2004. Actualités en réanimation préhospitalière. SFEM Efs, Paris, pp 87-158 